\title{
Distributed Cooperative Routing Algorithms for Maximizing Network Lifetime
}

\author{
Charles Pandana, W. Pam Siriwongpairat, Thanongsak Himsoon, and K. J. Ray Liu \\ Electrical and Computer Engineering Department, University of Maryland, College Park.
}

\begin{abstract}
In this paper, we study the impact of cooperative routing for maximizing the network lifetime in sensor network applications. We assume nodes in the network are equipped with a single omnidirectional antenna and they perform cooperative transmission to achieve transmit diversity. We propose a joint cooperative transmission and energy aware routing algorithm to prolong the network lifetime. In contrast to the previous works, our approach uses the maximum lifetime power allocation, instead of minimum power allocation. Using the maximum lifetime power allocation, the cooperative nodes allocate their transmit power according to the channel condition and the residual energy in the nodes. Our proposed scheme combines the maximum lifetime power allocation and the energy aware routing to maximize the network lifetime. We study the performance of the cooperative routing in terms of network lifetime (defined as the time until the first node dies) and the total delivered packets before the first node dies. We demonstrate that the proposed solution achieves $1 \sim 3.5$ and $1 \sim 2$ times longer network lifetime and more total delivered packets compared to noncooperative routing, when it is used with MTE and FA algorithms, respectively. Furthermore, the maximum lifetime power allocation achieves $1 \sim 2$ times longer lifetime, compared to minimum power allocation in MTE and FA routing schemes. We also provide distributed implementation of the proposed algorithm.
\end{abstract}

\section{INTRODUCTION}

Advances in low power integrated circuit devices and communications technologies have enabled the deployment of lowcost, low power sensors that can be integrated to form sensor networks. This sensor network has vast important applications and has been identified as one of the most important technologies nowadays. The deployment of the low cost and energy limited sensors implies that the energy efficient communication protocol is imperative to extend the lifetime of the network. The problem of energy efficient protocol can be approached from different communication layers; from physical layer, data-link layer, MAC layer, network layer to the application layer. Moreover, the cross layer approach has been shown to be an effective energy saving method in the energy constrained communication [1], [2]. In ad hoc networking environment, most of the energy consumption is due to the packet transmission [3]. Motivated by this fact, we focus on the cross layer approach by jointly design the energy efficient routing algorithm in network layer and the energy efficient signal combining in physical layer.

The energy efficient routing and transmit diversity have been studied separately in the literatures. The transmit diversity, pioneered by Alamouti's paper [4] shows the significant performance gain can be achieved in the multiple-input-multiple-output (MIMO) systems. However, multiple antennas in a sensor node may be impractical due to the cost. To overcome this problem, the cooperative communication concept has been recently proposed [5]. This cooperative communication explores the broadcast nature of the wireless medium, where signal transmitted by a node will be received by all nodes within its transmission range.
This property is usually referred to as the wireless broadcast advantage. In the multi-hop transmission, nodes that have received the transmitted signal will cooperatively help relaying and form a virtual multi antenna system. This virtual multi antenna system achieves significant performance gain as in the MIMO system.

There are many existing energy efficient routing algorithms in the literatures. The minimum total energy routing (MTE) [6] algorithm selects the route that minimizes the total transmission energy along the route. The min-max battery cost routing (MMBCR) algorithm [7] chooses the route to avoid the overuse of nodes along the minimum total energy route. However, the MMBCR route is far from the energy efficient route. To overcome the problem, [8] proposed a heuristic called flow augmentation (FA) algorithm that gradually makes transition from MTE to MMBCR. However, all existing energy efficient algorithms do not jointly utilize the cooperative transmission and energy efficient routing in performing routing decision. In [9], a joint cooperative communication and routing algorithm is proposed with the goal of minimizing the route energy.

In this paper, we propose a cooperative routing algorithm to maximize the network lifetime. The proposed scheme combines the cooperative transmission and the energy aware routing algorithm. To maximize the network lifetime, we propose maximum lifetime power allocation in the cooperative transmission. The maximum lifetime power allocation allocates transmit power in each node according to the channel condition and the normalized remaining energy in the node. We argue and demonstrate that using this criterion along with any energy aware routing algorithm (such as MTE and FA algorithms) results in longer network lifetime. We show the effectiveness of our proposed method through extensive simulations. The remaining of the paper is organized as follows. Section II describes the system model, reviews the existing energy aware routing algorithm and the cooperative link cost formulation. In Section III, we derive and explain the proposed maximum lifetime power allocation in cooperative transmission. We also discuss the joint cooperative transmission and energy aware routing scheme. The possible distributed implementation of the proposed cooperative routing is presented in Section IV. The performance of our proposed algorithm is evaluated in Section V. Finally, conclusions are drawn in Section VI.

\section{SySTEM MODEL}

We model the wireless network as an undirected simple finite graph $G(V, E)$, where $V$ is the set of nodes in the network and $E$ is the set of all links/edges. The link $(i, j) \in E$ implies that node $j \in S_{i}$ can be directly reached by node $i$ with a certain transmit power level in the predefined dynamic range, where $S_{i}$ is the set of nodes that can be directly reached by node $i$. We 
assume that every node has the initial battery energy of $\mathcal{E}_{i}$ for $\forall i \in V$. Every packet transmission consumes energy. The energy expenditure for transmission from node $i$ to $j$ is proportional to $d(i, j)^{\alpha}$, where $d(i, j)$ is the distance between node $i$ and $j, \alpha$ is between $2 \sim 4$ and it depends on the transmission environment [10]. In this paper, we assume $\alpha=2$ as the path loss exponent for free space propagation. In the rest of this section, we summarize the existing energy-aware routing algorithms and the link cost formulation in cooperative transmission.

\section{A. Energy-aware routing}

The MTE routing uses the transmission energy consumed in the node as the link cost between that node and its neighbors. If we consider a route $r=\left\{n_{0}, \cdots, n_{d}\right\}$, where $n_{0}$ is the source node and $n_{d}$ is the destination node. Let denote the energy consumed in transmitting packet over the hop $\left(n_{i}, n_{j}\right)$ as $e\left(n_{i}, n_{j}\right)$, then the total expended energy in that route is $P(r)=\sum_{i=0}^{d-1} e\left(n_{i}, n_{i+1}\right)$. The MTE routing selects the route among all routes that minimizes the total expended energy in the route

$$
r_{M T E}^{*}=\arg \min _{r \in R\left(n_{0}, n_{d}\right)} P(r),
$$

where $R\left(n_{0}, n_{d}\right)$ is the set of routes from source node $n_{0}$ to destination node $n_{d}$.

The FA algorithm is similar to the MTE routing algorithm, except it weighs the energy consumed over one hop by the normalized residual energy. In particular, the FA algorithm employs $e\left(n_{i}, n_{j}\right)^{x_{1}} \underline{E}_{n_{i}}^{-x_{2}} E_{n_{i}}^{x_{3}}$ as the energy metric over the hop $\left(n_{i}, n_{j}\right)$, where $\underline{E}_{n_{i}}$ is the residual energy of node $n_{i}$ at current time and $E_{n_{i}}$ is the initial energy of node $n_{i}$, and $x_{1}, x_{2}, x_{3}$ are parameters that govern the effect of normalized residual energy. Therefore, the total weighted energy expended in a route is $P(r)=\sum_{i=0}^{d-1} e\left(n_{i}, n_{i+1}\right)^{x_{1}} \underline{E}_{n_{i}}^{-x_{2}} E_{n_{i}}^{x_{3}}$. And the algorithm selects the route that minimizes the total weighted energy,

$$
r_{F A}^{*}=\arg \min _{r \in R\left(n_{0}, n_{d}\right)} \sum_{i=0}^{d-1} e\left(n_{i}, n_{i+1}\right)^{x_{1}} \underline{E}_{n_{i}}^{-x_{2}} E_{n_{i}}^{x_{3}} .
$$

This metric gradually avoids the minimum energy path as the residual energy of nodes along the minimum energy path is low. We use $\left(x_{1}, x_{2}, x_{3}\right)=(1,5,5)$ throughout this paper.

\section{B. Link cost formulation}

Employing the cooperative transmission changes the link cost for the routing algorithm. Let denote the transmitter set as $T_{x}=$ $\left\{t_{1}, \cdots, t_{n}\right\}$ and the receiver set as $R_{x}=\left\{r_{1}, \cdots, r_{m}\right\}$. We summarize the link cost in the following 4 transmission modes [9].

1. Point-to-point, $T_{x}=\left\{t_{1}\right\}, R_{x}=\left\{r_{1}\right\}$ : the received signal is represented as

$$
y(t)=\beta \omega s(t)+\eta(t),
$$

where $\beta=d\left(t_{1}, r_{1}\right)^{-\alpha / 2}$ is the channel response, $d\left(t_{1}, r_{1}\right)$ is the distance between node $t_{1}$ and $r_{1}, s(t)$ is the unit-energy transmit signal, and $\eta(t)$ is the additive gaussian noise. The transmit power at the transmitter is represented as $P=|\omega|^{2}$ and the SNR at the receiver side is $\gamma=\frac{\beta^{2}|\omega|^{2}}{P_{\eta}}$, where $P_{\eta}$ is the noise variance. We assume the receiver can correctly decode the received signal if the receiver side SNR is above the minimum threshold value, $\gamma_{\min }$. Therefore, the link cost in pointto-point transmission is described as

$$
L\left(t_{1}, r_{1}\right)=e\left(t_{1}, r_{1}\right)=\frac{\gamma_{\min } P_{\eta}}{\beta^{2}} .
$$

2. Broadcast, $T_{x}=\left\{t_{1}\right\}, R_{x}=\left\{r_{1}, \cdots, r_{m}\right\}$ : using the wireless broadcast advantage, the cost for reaching the receiver set $R_{x}$ is the cost to reach the farthest receiver, i.e.,

$$
L\left(t_{1}, R_{x}\right)=\max \left\{L\left(t_{1}, r_{1}\right), \cdots, L\left(t_{1}, r_{m}\right)\right\} .
$$

3. Cooperative, $T_{x}=\left\{t_{1}, \cdots, t_{n}\right\}, R_{x}=\left\{r_{1}\right\}$ : the signal at the receiver is

$$
y(t)=\sum_{i=1}^{n} \beta_{i 1}\left|\omega_{i}\right| s(t)+\eta(t)
$$

where $\beta_{i 1}=d\left(t_{i}, r_{1}\right)^{-\alpha / 2}$. Using simple calculation, the link cost/total power allocation can be obtained as

$$
L\left(T_{x}, r_{1}\right)=\frac{1}{\sum_{i=1}^{n} \frac{\beta_{i 1}^{2}}{\gamma_{\min } P_{\eta}}} .
$$

The energy consumption in each transmitter is presented as

$$
e\left(t_{1}, r_{1}\right)=\frac{\beta_{i 1}^{2} \gamma_{\min } P_{\eta}}{\left(\sum_{i=1}^{n} \beta_{i 1}^{2}\right)^{2}}
$$

4. Cooperative-Broadcast, $T_{x}=\left\{t_{1}, \cdots, t_{n}\right\}, R_{x}=$ $\left\{r_{1}, \cdots, r_{m}\right\}$ : If we assume perfect synchronization and channel estimation, the link cost for this mode of transmission will be

$$
L\left(T_{x}, R_{x}\right)=\max \left\{\frac{1}{\sum_{i=1}^{n} \frac{\beta_{i 1}^{2}}{\gamma_{\min } P_{\eta}}}, \cdots, \frac{1}{\sum_{i=1}^{n} \frac{\beta_{i m}^{2}}{\gamma_{\min } P_{\eta}}}\right\} .
$$

Suppose $m^{*}$ is the element with the highest link cost in (9), the energy consumption in each of the transmitter is

$$
e\left(t_{1}, R_{x}\right)=\frac{\beta_{i m^{*}}^{2} \gamma_{\min } P_{\eta}}{\left(\sum_{i=1}^{n} \beta_{i m^{*}}^{2}\right)^{2}}
$$

\section{PROposed SOLUTION}

\section{A. Maximum lifetime power allocation}

In this section, we propose a different power allocation that takes into account the goal of the routing algorithm; that is to maximize the network lifetime. We note that the flow augmentation routing algorithm minimizes the total transmit power in the route weighted by the normalized residual energy. By weighting the energy metric with the normalized residual energy, the route with extremely low residual node will be avoided. Based on this concept, we re-derive the power allocation problem in the cooperative transmission case and we refer the resulting 


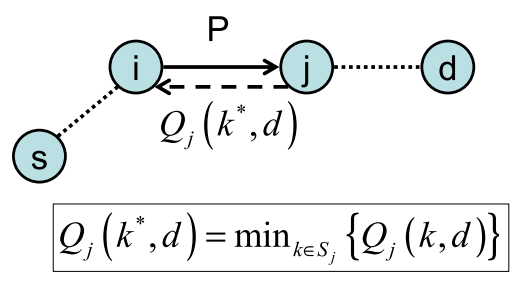

Fig. 1: Exchange and Update $Q$-value

power allocation to as the maximum lifetime power allocation. Specifically, we formulate the optimization problem as

$$
\begin{array}{ll}
\min & \sum_{i=1}^{n} \frac{E_{i}}{E_{i}}\left|\omega_{i}\right|^{2} \\
\text { s.t. } & \frac{\left|\sum_{i=1}^{n} \beta_{i 1} \omega_{i}\right|^{2}}{P_{\eta}} \geq \gamma_{\text {min }} .
\end{array}
$$

The above optimization problem minimizes the weighted total power while ensuring the received SNR is larger than minimum required SNR. Using the Lagrange multiplier method we have

$L\left(\omega_{1}, \cdots, \omega_{n}, \lambda\right)=\sum_{i=1}^{n} \frac{E_{i}}{\underline{E}_{i}}\left|\omega_{i}\right|^{2}-\lambda\left(\frac{\left|\sum_{i=1}^{n} \beta_{i 1} \omega_{i}\right|^{2}}{P_{\eta}}-\gamma_{\text {min }}\right)$,

Taking the partial derivatives, we have

$$
\begin{gathered}
\frac{\partial L}{\partial \omega_{i}}=2 \frac{E_{i}}{\underline{E}_{i}} \omega_{i}-2 \frac{\lambda \beta_{i 1}\left|\sum_{i=1}^{n} \beta_{i 1} \omega_{i}\right|}{P_{\eta}}=0, \forall i \\
\frac{\partial L}{\partial \lambda}=\frac{\left|\sum_{i=1}^{n} \beta_{i 1} \omega_{i}\right|^{2}}{P_{\eta}}-\gamma_{\text {min }}=0
\end{gathered}
$$

Equivalently, (13) can be represented as

$$
\omega_{i}=\frac{E_{i}}{E_{i}} \lambda \beta_{i 1} \frac{\sqrt{P_{\eta} \gamma_{\min }}}{P_{\eta}} .
$$

We note that we have use $\left|\sum_{i=1}^{n} \beta_{i 1} \omega_{i}\right|^{2}=P_{\eta} \gamma_{\text {min }}$ to get (15). Substituting (15) to (14), we get

$$
\sum_{i=1}^{n} \frac{\underline{E}_{i}}{E_{i}} \lambda \beta_{i 1}^{2} \frac{\sqrt{P_{\eta} \gamma_{\min }}}{P_{\eta}}=\sqrt{P_{\eta} \gamma_{\min }}
$$

Hence, we have

$$
\lambda=\frac{P_{\eta}}{\sum_{i=1}^{n}\left(\frac{E_{i}}{E_{i}}\right) \beta_{i 1}^{2}} .
$$

This implies that (15) is equivalent to

$$
\omega_{i}=\frac{\frac{\underline{E}_{i}}{\bar{E}_{i}} \beta_{i 1} \sqrt{P_{\eta} \gamma_{\min }}}{\sum_{i=1}^{n}\left(\frac{\underline{E}_{i}}{\bar{E}_{i}}\right) \beta_{i 1}^{2}},
$$

and the energy consumption of cooperative transmission in each node using maximum lifetime criterion is

$$
e\left(t_{i}, r_{1}\right)=\left|\omega_{i}\right|^{2}=\frac{\left(\frac{\underline{E}_{i}}{E_{i}}\right)^{2} \beta_{i 1}^{2} P_{\eta} \gamma_{\text {min }}}{\left[\sum_{i=1}^{n}\left(\frac{\underline{E}_{i}}{E_{i}}\right) \beta_{i 1}^{2}\right]^{2}} .
$$

This power allocation criterion (19) has the interpretation that in addition to using the channel condition $\beta_{i 1}$ for power allocation, the node who has abundant residual energy will help more

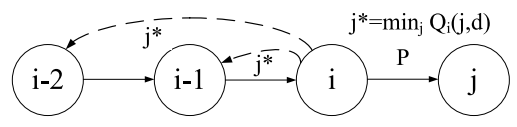

Fig. 2: Cooperation transmission illustrated

on the cooperative transmission compared to the node with less residual energy. In initial deployment, when all the sensor nodes have abundant of residual energy, the criterion (19) practically reduces to (8). We will show by simulation in Section $\mathrm{V}$, that the joint maximum lifetime power allocation and the maximum lifetime routing algorithm can significantly extend the network lifetime.

\section{B. Joint maximum lifetime routing and power allocation}

The simplest way to jointly consider the maximum lifetime routing and the maximum lifetime power allocation is to select the cooperating nodes along the noncooperative route [9]. The obvious pros of this method are that it is simple and it is very easy to design the distributed solution. The cooperative MTE and cooperative FA algorithm are summarized in Table I and Table II, respectively.

TABLE I: Centralized cooperative MTE- $n$

1. Find the minimum total energy route with edge cost as in (8)
2. Select the last $n$ nodes in the MTE route to do cooperative
transmission.
3a. For minimum energy allocation, deduct the amount of energy
proportional to (8) from each of cooperating nodes.
3b. For maximum lifetime energy allocation, deduct the amount
of energy proportional to (19) from each of cooperating nodes.

TABLE II: Centralized cooperative FA $\left(x_{1}, x_{2}, x_{1}\right)-n$

1. In every update time: find the maximum lifetime route with edge cost
between node $i$ and $j$ as $e(i, j)^{x_{1}} \underline{E}_{n_{i}}^{-x_{2}} E_{n_{i}}^{x_{3}}$. The optimal route is
denoted as $r^{*}=\arg \min _{r \in R(s, d)} \sum_{i=0}^{d-1} e\left(n_{i}, n_{i+1}\right)^{x_{1}} \underline{E}_{n_{i}}^{-x_{2}} E_{n_{i}}^{x_{3}}$.
2. Select the last $n$ nodes in the FA route to do cooperative transmission.
3a. For minimum energy allocation, deduct the amount of energy
proportional to (8) from each of cooperating nodes.
3b. For maximum lifetime energy allocation, deduct the amount
of energy proportional to (19) from each of cooperating nodes.

\section{Distributed COOPERATIVE ROUTING AND LEARNING}

In this section, we develop a distributed method to implement the maximum lifetime cooperative routing algorithm. The method is based on the distributed reinforcement learning routing algorithm [11]. The routing decision is learned by all nodes in the network. Each node maintains the best packet delivery cost to all the destinations. In particular, each node $i$ maintains a table of $Q$-values $Q_{i}(j, d)$, for $j \in S_{i}$, where $j$ is in the set of node $i$ neighbors, $S_{i}$, and node $d$ is the destination. The $Q_{i}(j, d)$ has the interpretation of node $i$ 's best estimated cost that a packet would incur to reach its destination node $d$ from node $i$ when the packet is sent via node $i$ 's neighbor node $j$.

The value in the $Q$-table will be exchanged between node $i$ and $j$, whenever there is a packet is sent from node $i$ and $j$, and vice versa. The exchange mechanism is illustrated as in Figure 1. Whenever node $i$ transmits a packet $P$ to node $j$, node $j$ feedbacks $Q_{j}\left(k^{*}, d\right)=\min _{k \in N(j)} Q_{j}(k, d)$ to node $i$ as shown in the figure. The node $i$ uses this value to update its own $Q$-value as follow

$$
Q_{i}(j, d)=(1-\delta) Q_{i}(j, d)+\delta\left[Q_{j}\left(k^{*}, d\right)+c(i, j)\right],
$$




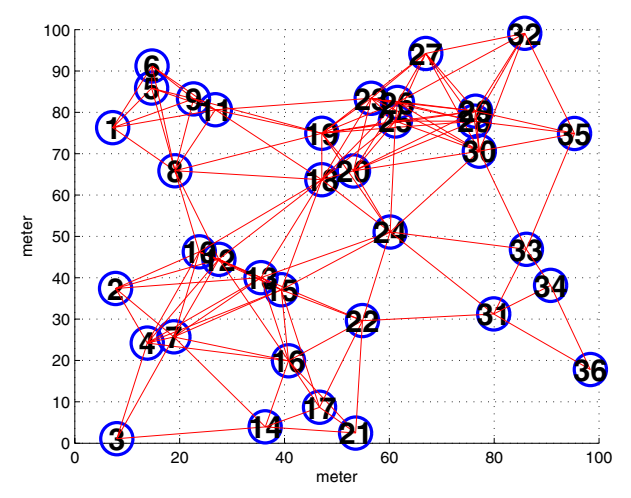

Fig. 3: Random network with 36 nodes in 100 meter by 100 meter area

where $c(i, j)$ is the cost for sending packet from node $i$ to node $j$, and $\delta \in[0,1]$ is the learning rate for the algorithm.

Since in this paper, the routing algorithms are driven for the purpose of maximizing the network lifetime, then the cost of sending a packet between node $i$ and node $j$ is related to the energy consumption for sending the packet. In particular, the cost of sending packet for MTE and FA routing algorithms are

$$
\begin{aligned}
{[\mathrm{MTE}]: } & c(i, j)=e(i, j), \\
{\left[\mathrm{FA}\left(x_{1}, x_{2}, x_{3}\right]:\right.} & c(i, j)=e(i, j)^{x_{1}} \underline{E}_{n_{i}}^{-x_{2}} E_{n_{i}}^{x_{3}},
\end{aligned}
$$

For MTE, $Q_{i}(j, d)$ represents the total energy consumption used to delivery a packet from node $i$ to node $d$ via node $i$ 's neighbor node $j$. In contrast, $Q_{i}(j, d)$ in FA represents the total energy consumption in delivering a packet from node $i$ to $d$ via $j$, weighted by the normalized residual energy of nodes along the route. We note that the entire route used for packet transmission is appended to the header when doing the learning. After making the next hop decision and before transmitting the packet, the node will inform its previous $n-1$ nodes to do the cooperation based on the route in the packet header. Figure 2 illustrates this situation where node $i$ has made the routing decision, but it has not transmitted the packet $P$ yet. At this time node $i$ informs the $n-1$ nodes to engage in cooperation ( $n=3$ is shown in the figure). In this way, the cooperative transmission also helps reducing the transmit energy expended during the learning period.

\section{Simulation Results}

We simulate the packet routing system as the discrete event system. The topology of the simulated network is shown in Figure 3 . The network consists 36 nodes, which uniformly deployed in an 100 meter by 100 meter area. The traffic is generated from node 21 to node 6 and node 32 to node 3 . The packet arrival follows the Poisson distribution and the number of packets introduced per unit simulation time step is referred to as packet arrival rate (traffic load), $\mu$. Multiple packets generated at a node are stored in its first-in-first-out queue. At every time step, each node removes the packet in front of its queue and sends the packet to the next hop according to the routing decision. When a node receives a packet, it either queues the received packet at the end of its queue or removes the packet from the network. The latter case happens when the packet arrives at its destination. All the nodes in the network initially have $\mathcal{E}_{i}=100, \forall i \in V \backslash\{21,32\}$ unit energy,

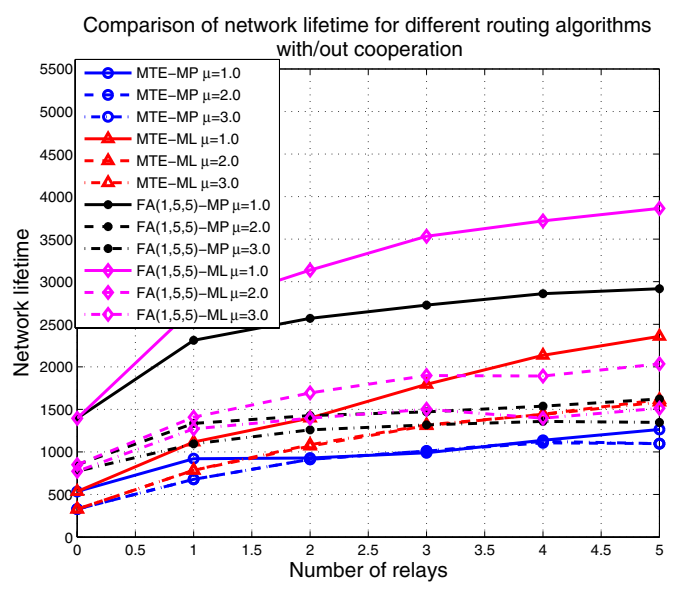

Fig. 4: Network lifetime

except node 21 and node 32 , which have $\mathcal{E}_{21}=1000$ and $\mathcal{E}_{32}=$ 1000 .

We compare 2 routing algorithms, namely MTE and FA algorithm, each with 2 power allocations. We referred the MTE with the minimum power allocation criterion (8) to as MTE-MP and the MTE with the maximum lifetime power allocation (19) to as MTE-ML. Similar naming is given for FA algorithm. We compare network lifetime, average packet delivery time, average energy per packet, and total packet delivery. The network lifetime (measured in terms of simulation time step) is defined as the time before the first node dies. The average packet delivery time is defined as the time between packet introduction at the source and its removal time at the destination. The average energy per packet is the total energy consumed per delivered packets. Finally, the total delivered packets is the number of packets that are successfully delivered before the first node dies.

Figure 4 shows the lifetime of the network that is achieved by different centralized routing algorithm with minimum power allocation and maximum lifetime power allocation. We note that for the centralized routing algorithm, the minimum total energy route is only calculated at the beginning of the simulation. In contrast, the FA algorithm recomputes the route every 20 simulation time step using the most current residual energy on each node. The performance of each scheme is compared for different traffic load, namely low load $(\mu=1.0)$, medium load $(\mu=2.0)$, and high load $(\mu=3.0)$. The $\mathrm{x}$-axis of the figure represents the number of relays used in the cooperative transmission. We recall that the $n$-relays are selected from the last $n$ nodes along the noncooperative MTE and FA algorithm, correspondingly. In the figure, the cooperative MTE-MP achieves $71.77 \%, 73.45 \%, 85.06 \%, 112.30 \%$, and $136.20 \%$, longer network lifetime when $1,2, \cdots, 5$ relays are used, respectively, compared to the noncooperative routing. However, compared to the noncooperative routing, the MTE-ML achieves $108.72 \%$, $161.05 \%, 235.09 \%, 298.88 \%$, and $340.54 \%$, longer network lifetime when $1,2, \cdots, 5$ relays are used. Obviously, we see that the maximum lifetime power allocation achieves much higher network lifetime compared to the maximum power allocation. The MTE-ML algorithm achieves from $1 \sim 3.5$ times higher network lifetime compared to noncooperation routing. Compared 


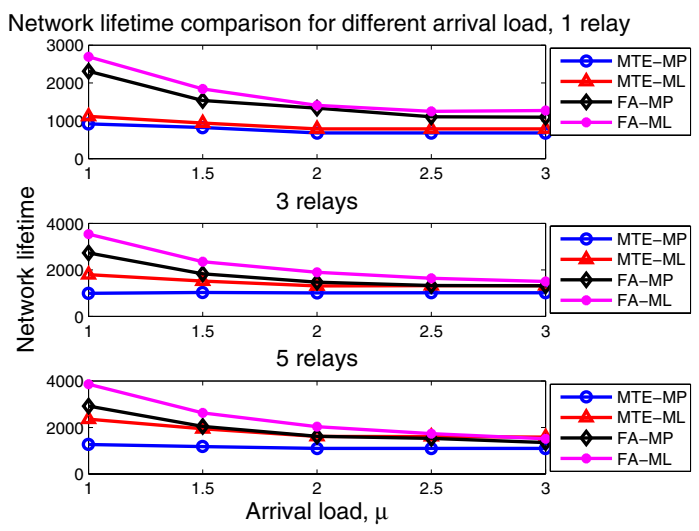

Fig. 5: Network lifetime comparison for different routing algorithms when the number of relays is 1,3 , and 5

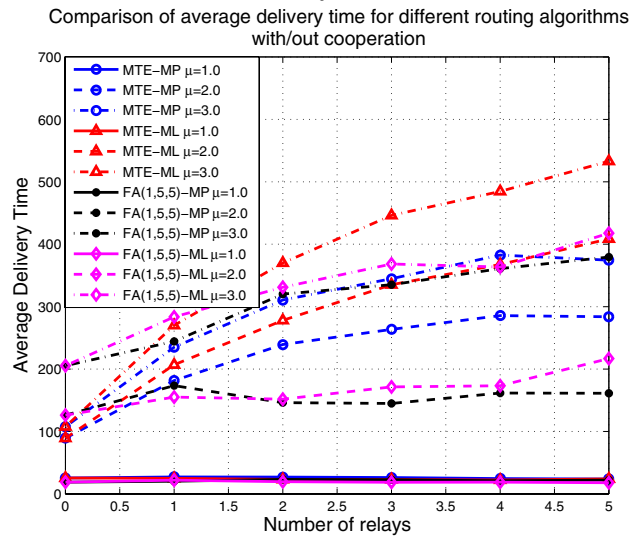

Fig. 6: Average delivery time

to MTE-MP, MTE-ML achieves around $1 \sim 2$ times better network lifetime, when $1 \sim 5$ relays are used. The reason for this phenomenon is that the minimum power allocation allocates the power merely based on the channel condition (in our model (8) farther away nodes have lower channel gain, due to path loss, and therefore allocate less transmit power). Hence, in some popular route (minimum total energy route), some nodes will be overused in the minimum power allocation and the time until the first node dies (network lifetime) is shorter. In contrast, in maximum lifetime power allocation, the power allocation allocates the power according to the normalized residual energy in the nodes and the channel condition. Therefore, the situation that one particular node is overused will be minimized in cooperative transmission and MTE-ML results in longer network lifetime. Figure 4 also shows the network lifetime when the FA algorithm is used. Compared to the noncooperative routing, the FA-MP achieves $65.72 \%$, $84.29 \%, 95.45 \%, 105.01 \%$, and $109.18 \%$ longer lifetime, when $1,2, \cdots, 5$ relays are used, respectively. In contrast, the FA-ML achieves $93.02 \%, 124.89 \%, 153.44 \%, 166.41 \%$, and $176.96 \%$, when $1,2, \cdots, 5$ relays are used, respectively.

Figure 5 shows the sensitivity of the routing algorithm to the network load. From this figure, we observe that the MTE-MP is the least sensitive to the traffic load among all the algorithms. This can be understood since the MTE-MP only uses the minimum total transmit power as the routing selection and power allocation criterion. No matter how the traffic arrival load is, the route

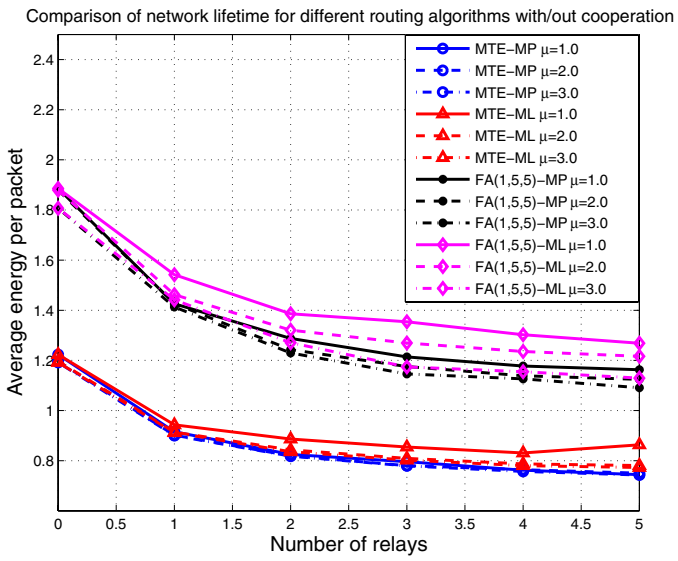

Fig. 7: Average consumed energy per packet

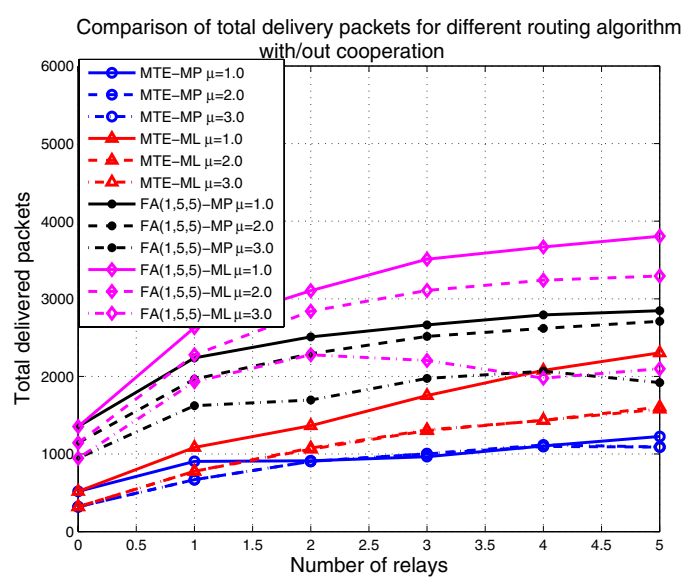

Fig. 8: Total delivered packets

selection and power allocation will not change. However, the MTE-ML, FA-MP, and FA-ML algorithms choose the route and power allocation according to the normalized residual energy in the nodes. When the traffic arrival load is small, the ML algorithm tries its best to balance the load to all the cooperative nodes. Similarly, the FA algorithm tries to balance the load to nodes in all possible routes between the source and destination. As the traffic load becomes large, the algorithms find less flexibility to distribute the load among either the cooperative nodes or the nodes in routes between source and destination. Hence, the performance gain of algorithms based on normalized residual energy becomes smaller as the arrival load becomes larger. In all cases, the FA-ML is superior to FA-MP, FA-MP outperforms MTE-ML, and MTEML is better than MTE-MP. But, the performance gains become smaller as the network arrival load is larger.

Figures 6-8 show the average delivery time, average consumed energy per packet and the total delivery packets before the first node dies. In Figure 6, all the algorithms have similar delivery time when the traffic load is low $(\mu=1.0)$. In the medium traffic load, the FA algorithms with MP and ML power allocation have lower delivery time compared to MTE algorithm. This is due to the fact that FA algorithm explores several routes besides the minimum energy route and distributes the traffic to queues among routes between source and destination. Therefore, the 


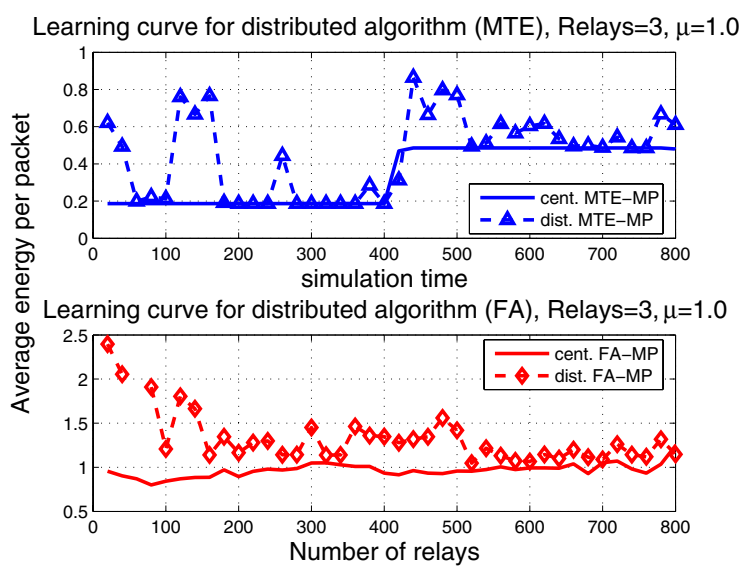

Fig. 9: Learning curves in the distributed learning algorithm algorithm has practically lower delivery time. However, in the large network load, all the algorithms except MTE-ML have similar delivery time. Figure 7 shows the average energy per packet consumed by different routing algorithms. It is obvious that the MTE class of the algorithm has the lower energy consumed per packet. The FA algorithm has higher energy consumption because the algorithm selects less energy efficient route to balance the energy consumption among nodes. In both algorithms, the cooperative transmission is very effective in lowering the energy consumed per packet. Finally, Figure 8 shows the number of packets successfully delivered before the first node dies. The performance of different routing algorithms in this metric is very similar to the network lifetime. The FA-ML outperforms FA-MP, FA-MP outperforms MTE-ML, and MTE-ML is better than MTEMP in low to high traffic load.

Figures 10 and 9 show the distributed reinforcement learning implementation for all routing algorithms according to Section IV. In this simulation, the learning parameter is chosen as $\delta=0.85$. Figure 9 shows the learning curves in distributed implementation when the traffic load is 1.0 and 3 cooperative relay nodes are used. It is obvious that the distributed MTE-MP converges to the centralized solution within 100 simulation time. In contrast, the distributed FA algorithm converges to the centralized solution in rather longer time. We recall that the optimal route according to the FA algorithm changes as the residual energy in nodes changes. In this time varying route selection, the distributed implementation is able to achieve the centralized solution. Finally, Figure 10 shows the result of learning algorithm for different routing algorithms. In this figure, the distributed implementation achieves almost similar in network lifetime to the centralized solution. However, if we observe the total delivered packet, the distributed algorithm achieves much lower throughput compared to the centralized solution. The reason for this is that the distributed algorithms consume some portion of the nodes' energy to explore and learn the good routing decision. During the exploration (learning) stage, loops in the route may appear and the algorithms practically deliver only a small portion of the traffic. From the figure, one can observe that the cooperative transmission still improves the number of delivered packet.

\section{CONCLUSIONS}

We have proposed an effective way to do the power allocation in cooperative transmission. In the cooperative routing, the power

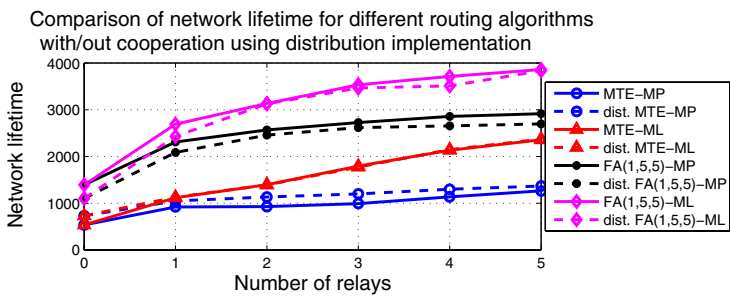

Comparison of network lifetime for different routing algorithms

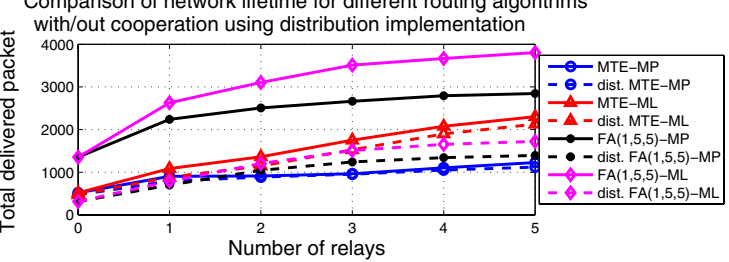

Fig. 10: Comparison of network lifetime and total delivery packets for distributed reinforcement learning implementation $\mu=1.0$

allocation and the routing decision should be jointly decided to maximize the network lifetime. To achieve maximum network lifetime, the proposed scheme employs the maximum lifetime power allocation and the energy aware routing. The maximum lifetime power allocation allocates transmit power according to each node's channel condition and its residual energy in cooperative transmission. This criterion results in a situation where nodes with more residual energy help more compared to nodes with less energy. Compared to the minimum power allocation, our proposed method achieves $1 \sim 2$ longer network lifetime and more total delivered packets in MTE algorithm and FA algorithm. We also outline the distributed implementation of the algorithms and show that the distributed algorithm converges to the centralized route.

\section{REFERENCES}

[1] A. J. Goldsmith and S. B. Wicker, "Design challenges for energy-constrained ad hoc wireless networks ", in IEEE Wireless Communications, vol. 9, August 2002 , pp. 827.

[2] C. Pandana and K. J. Ray Liu, "Near-optimal reinforcement learning framework for energy-aware sensor communications", in IEEE Journal on Selected Areas in Communications, vol. 23, April 2005, pp. 788 - 797.

[3] Laura Marie Feeney and Martin Nilsson, "Investigating the energy consumption of a wireless network interface in an ad hoc networking environment", in Proc. IEEE INFOCOM, vol. 3, 22-26 April 2001, pp.1548 - 1557.

[4] S. M. Alamouti, "A simple transmit diversity technique for wireless communications", in IEEE Journal on Selected Areas in Communications, vol. 16, Oct. 1998, pp.1451-1458.

[5] J. N. Laneman, D. N. C. Tse, and G. W. Wornell, "Cooperative diversity in wireless networks: efficient protocols and outage behavior", in IEEE Transactions on Information Theory, vol. 50, December 2004, pp. 3062-3080.

[6] C.-K. Toh, "Maximum battery life routing to support ubiquitous mobile computing in wireless ad hoc networks", in IEEE Communications Magazine, June 2001, pp. 138-147.

[7] S. Singh, M. Woo and C. S. Raghavendra, "Power-aware routing in mobile ad hoc networks", in Proc. 4th Annual IEEE/ACM Int. Conf. Mobile Computing and Networking, Dallas, TX, Oct. 1998, pp. 181-190.

[8] J.-H. Chang and L. Tassiulas, "Maximum lifetime routing in wireless sensor networks", in IEEE/ACM Transactions on Networking, vol. 12, no. 4, Aug. 2004, pp. 609-619.

[9] Amir Ehsan Khandani, Jinane Abounadi, Eytan Modiano, and Lizhong Zheng, "Cooperative Routing in Wireless Networks", in Allerton Conference on Communications, Control and Computing, October, 2003, pp. 1270-1279.

[10] T. S. Rappaport, Wireless Communications: Principle and Practice Prentice Hall, July 1999.

[11] Michael L. Littman and Justin A. Boyan, "A distributed reinforcement learning scheme for network routing ", in Advances in Neural Infromation Processing Systems, vol. 6, 1993, pp.670-678. 